\title{
On interpolation of cocompact imbeddings
}

\author{
Michael Cwikel · Kyril Tintarev
}

Received: 31 May 2011 / Accepted: 15 October 2011 / Published online: 8 November 2011

(C) The Author(s) 2011. This article is published with open access at Springerlink.com

\begin{abstract}
Cocompactness is a useful weaker counterpart of compactness in the study of imbeddings between function spaces. In this paper we prove that, under quite general conditions, cocompactness of imbeddings of Banach spaces persists under both real and complex interpolation. As an application, we obtain that subcritical continuous imbeddings of fractional Sobolev spaces and Besov spaces are cocompact relative to lattice shifts. We deduce this by interpolating the known cocompact imbeddings for classical Sobolev spaces ("vanishing" lemmas of Lieb and Lions). We also apply cocompactness to prove compactness of imbeddings of some radial subspaces and to show the existence of minimizers in some isoperimetric problems. Our research complements a range of previous results, and recalls that there is a natural conceptual framework for unifying them.
\end{abstract}

Keywords Besov spaces · Cocompact imbeddings · Concentration compactness · Fractional Sobolev spaces $\cdot$ Interpolation spaces $\cdot$ Minimizers $\cdot$ Mollifiers $\cdot$ Sobolev imbeddings

Mathematics Subject Classification (2000) Primary 46B70 • 46E35 - 46B50 • Secondary $30 \mathrm{H} 25 \cdot 46 \mathrm{~N} 20 \cdot 49 \mathrm{~J} 45$

The research was supported by the Technion V.P.R. Fund and by the Fund for Promotion of Research at the Technion. Parts of this research have been conducted during visits of the authors to each other's home institutions.

M. Cwikel

Department of Mathematics, Technion-Israel Institute of Technology, Haifa 32000, Israel e-mail: mcwikel@math.technion.ac.il

url: http://www.math.technion.ac.il/ mcwikel/

K. Tintarev $(\bowtie)$

Department of Mathematics, Uppsala University, P.O. Box 480, 75106 Uppsala, Sweden e-mail: tintarev@math.uu.se url: http://tintarev.@comxa.com 


\section{Introduction}

The notion of cocompact imbedding is a convenient way to express a property of imbeddings, related to (and weaker than) compactness. Several authors, including Elliott Lieb, Pierre-Louis Lions and Terence Tao, have proved and used cocompactness of imbeddings of Sobolev and Strichartz spaces into $L^{p}$-spaces, without explicitly using this terminology. Although these and other results have long been perceived to be related to each other in the heuristic sense of concentration compactness, a formal and unified interpretation of them in functional analytic terms appeared only relatively recently.

Our starting point is the classical definition of a cocompact manifold: a manifold $M$ is called cocompact relative to a given group $G$ of automorphisms $\eta: M \rightarrow M$ if there exists a compact subset $K \Subset M$ such that for each $x \in M$ there exists some $\eta \in G$ such that $\eta x \in K$. In particular this implies that, for any sequence $\left\{x_{k}\right\}_{k \in \mathbb{N}}$ in $M$, there exists a sequence $\left\{\eta_{k}\right\}_{k \in \mathbb{N}}$ in $G$ such that $\left\{\eta_{k} x_{k}\right\}_{k \in \mathbb{N}}$ has a convergent subsequence in $M$.

When we seek to introduce a related notion in the context of Banach spaces, it turns out to be natural to do this in terms of the following modified version of weak convergence, defined relative to some group of continuous linear bijections.

Definition 1.1 Let $A$ be a Banach space, and let $\mathcal{D}$ be a group of continuous linear bijections of $A$. A sequence $\left\{u_{k}\right\}_{k \in \mathbb{N}}$ of elements of $A$ is said to be $\mathcal{D}$-weakly convergent to $u \in A$ (denoted by writing $u_{k} \stackrel{\mathcal{D}}{\rightarrow} u$ ), if $g_{k}\left(u_{k}-u\right) \rightarrow 0$ for all choices of the sequence $\left\{g_{k}\right\}_{k \in \mathbb{N}}$ in $\mathcal{D}$.

Note that since $\mathcal{D}$ contains the identity operator $I$, any $\mathcal{D}$-weakly convergent sequence is also weakly convergent. The converse is true if $\mathcal{D}$ is finite.

We can now state our main definition:

Definition 1.2 Let $A$ and $B$ be Banach spaces such that $A$ is continuously imbedded into $B$. Let $\mathcal{D}$ be a group of continuous linear bijections on $A$. We say that the imbedding of $A$ into $B$ is cocompact relative to $\mathcal{D}$ if every $\mathcal{D}$-weakly convergent sequence $\left\{u_{k}\right\}_{k \in \mathbb{N}}$ in $A$ converges in $B$.

Remark 1.3 There is a slightly different version of Definition 1.1 in [40] where $\mathcal{D}$ is merely a set, not necessarily a group, and all its elements are bounded linear operators. Definition 1.2 first appeared in [39], in fact in a marginally more restricted version where $\mathcal{D}$ is a group of linear isometries.

For later use we record the following elementary result which follows immediately from Definition 1.2.

Proposition 1.4 Let $X_{1}, X_{2}$ and $X_{3}$ be three Banach spaces with continuous imbeddings $X_{1} \subset X_{2} \subset X_{3}$. Suppose that the group $\mathcal{D}$ of linear operators $g: X_{3} \rightarrow X_{3}$ acts isometrically from $X_{j}$ to $X_{j}$ for $j=1,2,3$. Then the imbedding $X_{1} \subset X_{3}$ is $\mathcal{D}$-cocompact whenever at least one of the imbeddings $X_{1} \subset X_{2}$ and $X_{2} \subset X_{3}$ is $\mathcal{D}$-cocompact. 
Remark 1.5 When, in the context of Definition $1.2, \mathcal{D}=\{I\}$, and $A$ is reflexive, then of course cocompact imbeddings are simply compact imbeddings.

Remark 1.6 The notion of cocompactness facilitates the formulation of many results which can be considered as extensions of the Banach-Alaoglu theorem and which have wide applications. For example, an abstract version of such a result, in the context of Hilbert spaces due to Schindler and the second author of this paper is given in [31] and also appears as Theorem 3.1 of [40] pp. 62-63. Other versions, most of them in the setting of particular function spaces, and some of them for particular sequences, have been obtained (often independently) by a number of authors. Various terminologies, such as splitting lemmas, profile decompositions, intermediate topology, défaut de compacité, mutual divergence, dislocations or rescalings, vanishing sequences, asymptotic orthogonality, etc. have been used by a number of authors to describe the phenomena encountered in these results.

We expect that proceedings [2] of a forthcoming conference will provide a comprehensive view of the field.

The abstract Hilbert space version (i.e. the above-mentioned result in [31]) of this refinement of the Banach-Alaoglu theorem states that, in the presence of a suitably chosen group of operators $\mathcal{D}$ acting on a Hilbert space $A$, every bounded subsequence in $A$ has a subsequence of the following special structure: Each term in the subsequence is the sum of a principal term and a remainder term. The remainder terms form a sequence which converges $\mathcal{D}$-weakly, and each principal term is a (possibly infinite) sum of "dislocated profiles", i.e. terms of the form $g_{k} w$ where $g_{k} \in \mathcal{D}$ and $w \in A$.

Although no general analogue of this result is known for the case where $A$ is an arbitrary Banach space, the other results alluded to at the beginning of this remark are all of the same form, for suitable particular choices of $A$ and $\mathcal{D}$. The above-mentioned term, profile decomposition, is the one most commonly used for the special subsequence provided by such results.

The practical value of profile decompositions depends on finding some concrete space $B$ where the remainder sequence tends to zero in the norm. This is precisely what can be assured in those cases where the imbedding of $A$ into $B$ is known to be cocompact.

In an early cocompactness result about classical Sobolev imbeddings, $\mathcal{D}$ is taken to be the group $\mathcal{D}_{\mathbb{R}^{N}}$ of shifts $u \mapsto u(\cdot-y)$. This result is essentially due to Lieb [20] Lemma 6 on p. 447. In fact Lieb showed that any $\mathcal{D}_{\mathbb{R}^{N}}$-weakly convergent sequence in $W^{1, p}\left(\mathbb{R}^{N}\right)$ converges in measure, from which one can easily conclude that subcrit-

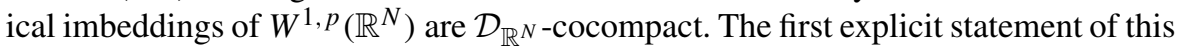
latter result is due, independently, to P.-L. Lions [23] Lemma I.1 on p. 231, which we restate below as Theorem 2.4. In the paper [26] Lions showed the existence of a profile decomposition for a specific sequence in $W^{1, p}\left(\mathbb{R}^{N}\right)$, again with $\mathcal{D}=\mathcal{D}_{\mathbb{R}^{N}}$. This result gave a more detailed description of the "loss of compactness" for that sequence than had been shown in his celebrated papers [22-25] on concentration compactness. The first proof of the existence of a profile decomposition for an arbitrary bounded sequence in the homogeneous Sobolev space $\dot{W}^{1, p}\left(\mathbb{R}^{N}\right)$ was given by Solimini [34] for the case where $\mathcal{D}$ is the product group of the actions of translations and dilations 
on $\mathbb{R}^{N}$. It is easy to deduce the existence of the particular profile decomposition in [26] from the result of [34]. Subsequently Gérard [15] gave an independent proof of a slightly weaker version of Solimini's result for the same group $\mathcal{D}$, but for somewhat different function spaces, namely $\dot{W}^{s, p}\left(\mathbb{R}^{N}\right)$ with $0<s<N / p$ and $p=2$. Gérard's result was extended by Jaffard [17] to all $p \in(1, \infty)$. Independently, the second author of this paper obtained similar results (Chap. 9 [40]) some portion of them jointly with Fieseler or Schindler, where $\mathbb{R}^{N}$ is replaced by a cocompact Riemannian manifold, an arbitrary nilpotent stratified Lie group, or a fractal blowup, with appropriate choices of $\mathcal{D}$. Additional references to other related works will be given in [2]. Among the most recent developments we mention the papers [37, 38], in which Tao, Visan and Zhang have proved the cocompactness of a Strichartz imbedding for the time-dependent Schrödinger equation, and also the work of Koch [19] where results in the style of $[15,17]$ are presented for embeddings of $L^{p}$ spaces into homogeneous Besov spaces with negative index of smoothness.

The main result of this paper, Theorem 2.3, deals with persistence of cocompactness for interpolated spaces. It can be considered as a sort of counterpart to results about persistence of compactness for operators mapping between "real method" or "complex method" interpolation spaces, in particular those in Sect. 9.6 of [9] and in [30], in which hypotheses having a partial analogy with hypotheses of Theorem 2.3 are imposed.

Remark 1.7 Note however that the compactness results of [9] and [30] were subsequently found to hold also without these kinds of hypotheses and/or under other alternative hypotheses. (See e.g. [10-14] and the references therein.) An analogous complete removal of additional conditions in the case of cocompactness would mean that persistence of cocompactness under interpolation holds for all choices of the group $\mathcal{D}$, which remains an open question. A negative answer to it would not surprise us.

As examples of applications of Theorem 2.3, we prove the cocompactness of classical Peetre imbeddings [27] of inhomogeneous Sobolev spaces with fractional indices of smoothness into $L^{p}$ spaces, relative to the group $\mathcal{D}_{\mathbb{Z}^{N}}$ of lattice shifts $u \mapsto u(\cdot-y)$ with $y \in \mathbb{Z}^{N}$. This is done in Theorem 2.5. Analogous results for imbeddings of Besov spaces are given in Theorems 2.6 and 2.8. These latter results can be thought of as variants of the results mentioned above of Gérard, Jaffard and Koch. In some ways they are not as sharp. On the other hand, unlike their results, ours deal with the case of inhomogeneous spaces.

Our results are stated in Sect. 2. Almost all of their proofs are deferred to subsequent sections. In Sect. 3 we give the proof of Theorem 2.3. Proofs of Theorem 2.5 and of Theorems 2.6 and 2.8 are provided in Sects. 4 and 5 respectively. We conclude our paper with Sect. 6 presenting two applications: We prove the compactness of certain imbeddings for subspaces of radial functions and the existence of minimizers for some isoperimetric problems involving fractional Sobolev spaces.

This paper lies at the intersection of two fields, the analysis of Sobolev spaces, and the theory of interpolation spaces. Since some readers may be more familiar with one of these fields than the other, we have taken the liberty of summarizing some of the 
basic notions from both of them, partly in the main body of the paper, and partly in appendices. In particular, in Appendix A, we recall definitions and results which we need from interpolation space theory, and in Appendix B we provide a version of the Brezis-Lieb lemma.

\section{Statements of the main results}

In all that follows, whenever we deal with Banach spaces, whose elements are functions $u: \mathbb{R}^{N} \rightarrow \mathbb{C}$ and whose norms are translation invariant, we will always choose the group $\mathcal{D}$ of Definitions 1.1 and 1.2 to be the set of lattice shifts. In other words, we take

$$
\mathcal{D}=\mathcal{D}_{\mathbb{Z}^{N}}:=\left\{g_{y}\right\}_{y \in \mathbb{Z}^{N}} \quad \text { where } g_{y} u=u(\cdot-y) .
$$

Whenever we deal here with a Banach couple $\left(A_{0}, A_{1}\right)$ we will always associate a group $\mathcal{D}$ to that couple, and the elements $g$ of $\mathcal{D}$ will always be assumed to be linear operators $g: A_{0}+A_{1} \rightarrow A_{0}+A_{1}$, such that

$$
g\left(A_{j}\right) \subset A_{j} \quad \text { and } \quad g: A_{j} \rightarrow A_{j} \quad \text { is an isometry for } j=0,1 .
$$

There are several frequently used different equivalent norms for the interpolation spaces $\left(A_{0}, A_{1}\right)_{\theta, p},\left[A_{0}, A_{1}\right]_{\theta}$ and for $A_{0}+A_{1}$. Here we will always use the standard norms whose definitions are recalled in Appendix A.

Lemma 2.1 Let $\left(A_{0}, A_{1}\right)$ be a Banach couple and let $\mathcal{D}$ be a group of linear maps $g: A_{0}+A_{1} \rightarrow A_{0}+A_{1}$ satisfying (2.2). Then each $g \in \mathcal{D}$ is also an isometry on $A_{0}+A_{1}$. Moreover, for every $p \in[1, \infty), \theta \in(0,1)$, the restriction of $g$ to $\left(A_{0}, A_{1}\right)_{\theta, p}$, respectively $\left[A_{0}, A_{1}\right]_{\theta}$, is an isometry on $\left(A_{0}, A_{1}\right)_{\theta, p}$, respectively $\left[A_{0}, A_{1}\right]_{\theta}$.

Proof This follows immediately from the basic interpolation properties of the spaces $\left(A_{0}, A_{1}\right)_{\theta, p}$ and $\left[A_{0}, A_{1}\right]_{\theta}$ and $A_{0}+A_{1}$ applied for the operators $g$ and $g^{-1}$.

We now introduce a definition of an operator family whose properties (i) and (ii) below are reminiscent of various conditions imposed to obtain interpolation of compactness in Sect. 9.6 of [9] and in [30]. As we shall see below, the standard mollifiers in Sobolev spaces, equipped with lattice shifts, are an example of a family of operators $M_{t}$ satisfying the definition.

Definition 2.2 Let $\left(A_{0}, A_{1}\right)$ be a Banach couple with $A_{1}$ continuously imbedded in $A_{0}$ and let $\mathcal{D}$ be a group of linear operators $g: A_{0}+A_{1} \rightarrow A_{0}+A_{1}$ which satisfies (2.2). Let $A_{1}$ be continuously imbedded into some Banach space $B_{1}$. A family of bounded operators $\left\{M_{t}\right\}_{t \in(0,1)}$ from $A_{0}$ to $A_{1}$ is said to be a family of $\mathcal{D}$-covariant mollifiers (relative to a space $B_{1}$ ) if it satisfies the following conditions:

(i) For $j=0,1$, the norm of $M_{t}$ as a continuous map from $A_{j}$ into itself is bounded independently of $t \in(0,1)$, i.e., $\sup _{t \in(0,1)}\left\|M_{t}\right\|_{A_{j} \rightarrow A_{j}}<\infty$. 
(ii) The function $\sigma(t):=\left\|I-M_{t}\right\|_{A_{1} \rightarrow B_{1}}$ satisfies $\lim _{t \rightarrow 0} \sigma(t)=0$.

(iii) For each $g \in \mathcal{D}$, and $t \in(0,1)$, there exists an element $h_{g, t} \in \mathcal{D}$ such that $g M_{t}=M_{t} h_{g, t}$.

Our main result is expressed in terms of general Banach couples.

Theorem 2.3 Let $\left(A_{0}, A_{1}\right)$ and $\left(B_{0}, B_{1}\right)$ be Banach couples with $A_{j}$ continuously imbedded in $B_{j}$ for $j=0,1$. Suppose, further, that $A_{1}$ is continuously imbedded in $A_{0}$. Let $\mathcal{D}$ be a group of linear operators $g: B_{0}+B_{1} \rightarrow B_{0}+B_{1}$ which satisfies (2.2) with respect to both of the couples $\left(A_{0}, A_{1}\right)$ and $\left(B_{0}, B_{1}\right)$. Assume that there exists a D-covariant mollifier family $\left\{M_{t}: A_{0} \rightarrow A_{1}\right\}_{t \in(0,1)}$. (See Definition 2.2.) If, furthermore, $A_{1}$ is $\mathcal{D}$-cocompactly imbedded into $B_{1}$, then, for every $\theta \in(0,1)$ and $q \in[1, \infty]$, the space $\left(A_{0}, A_{1}\right)_{\theta, q}$ is $\mathcal{D}$-cocompactly imbedded into $\left(B_{0}, B_{1}\right)_{\theta, q}$ and the space $\left[A_{0}, A_{1}\right]_{\theta}$ is $\mathcal{D}$-cocompactly imbedded into $\left[B_{0}, B_{1}\right]_{\theta}$.

We shall apply Theorem 2.3 to obtain cocompactness of interpolated imbeddings between certain function spaces. Our point of departure for doing this is the following cocompactness property of Sobolev imbeddings. It can be immediately shown to be an equivalent reformulation of results in papers [20] of Lieb and [23] of Lions. (See Lemma 6 on p. 447 of [20] and Lemma I.1 on p. 231 of [23].)

Theorem 2.4 Suppose that $p \in(1, \infty)$. The Sobolev imbedding of $W^{1, p}\left(\mathbb{R}^{N}\right)$ into $L^{q}\left(\mathbb{R}^{N}\right), p<q<p^{*}$, where $p^{*}=\frac{p N}{N-p}$ for $N>p$ and $p^{*}=\infty$ otherwise, is $\mathcal{D}_{\mathbb{Z}^{N}}$ cocompact.

In the following elementary application of Theorem 2.3, we shall extend this property to the Sobolev imbedding of the spaces $W^{\alpha, p}\left(\mathbb{R}^{N}\right)$ for all $\alpha \in(0, \infty)$. We recall one of the equivalent definitions of the space $W^{\alpha, p}\left(\mathbb{R}^{N}\right)$, namely as the space of all functions $f: \mathbb{R}^{N} \rightarrow \mathbb{R}$ in $L^{p}\left(\mathbb{R}^{N}\right)$ whose Fourier transforms $\widehat{f}$ are such that $\left(1+|\xi|^{2}\right)^{\alpha / 2} \widehat{f}(\xi)$ is also the Fourier transform of a function in $L^{p}\left(\mathbb{R}^{N}\right)$. This definition is valid for all real values of $\alpha>0$, including non integer values.

We recall the Sobolev-Peetre imbedding theorem, which states that the continuous inclusion $W^{\alpha, p}\left(\mathbb{R}^{N}\right) \subset L^{q}\left(\mathbb{R}^{N}\right)$ holds whenever $\alpha$ is positive and $1<p \leq q \leq p_{\alpha}^{*}$, where the critical exponent $p_{\alpha}^{*}$ is defined by

$$
p_{\alpha}^{*}= \begin{cases}\frac{p N}{N-\alpha p}, & N>\alpha p \\ \infty, & N \leq \alpha p .\end{cases}
$$

When $\alpha=1$ the prevalent notation is to write $p^{*}$ instead of $p_{1}^{*}$ (as we did just above in Theorem 2.4).

Theorem 2.5 Suppose that $\alpha \in(0, \infty)$ and $p \in(1, \infty)$. The Sobolev-Peetre imbedding of $W^{\alpha, p}\left(\mathbb{R}^{N}\right)$ into $L^{q}\left(\mathbb{R}^{N}\right)$ is $\mathcal{D}_{\mathbb{Z}^{N}}$-cocompact whenever $p<q<p_{\alpha}^{*}$. Moreover,

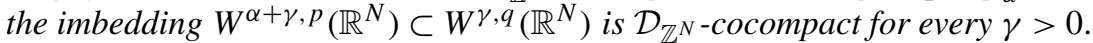

We now state our third result, which is obtained by applying Theorem 2.3 to couples of Sobolev spaces, for which the real interpolation method yields Besov spaces. 
(Relevant definitions are recalled in Appendix A.) The continuity of the imbeddings considered in this theorem is due to Jawerth [18].

Theorem 2.6 Suppose that $0<\beta<\alpha<\infty$ and $1<p_{0}<p_{1}<\infty$ and $q \in[1, \infty]$. If $\frac{N}{p_{0}}-\frac{N}{p_{1}}<\alpha-\beta$, then the continuous imbedding of $B^{\alpha, p_{0}, q}\left(\mathbb{R}^{N}\right)$ into $B^{\alpha, p_{1}, q}\left(\mathbb{R}^{N}\right)$ is $\mathcal{D}_{\mathbb{Z}^{N}}$-cocompact.

Corollary 2.7 Let $\alpha, \beta, p_{0}, p_{1}$ and $N$ be as in Theorem 2.6. Then the imbedding of

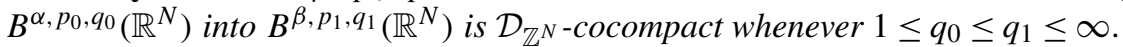

This corollary follows immediately from Proposition 1.4. We take $X_{1}=B^{\alpha, p_{0}, q_{0}}$,

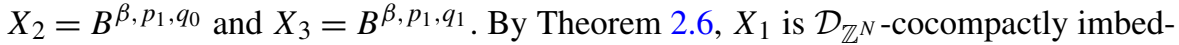
ded into $X_{2}$. The continuous imbedding $X_{2} \subset X_{3}$ follows from (6.17) and (6.8).

Theorem 2.8 Let $s>0,1<p<\infty, p<q_{0} \leq q<p_{s}^{*}$. Then the imbedding of $B$ s,p,q0 $\left(\mathbb{R}^{N}\right)$ into $L^{q}\left(\mathbb{R}^{N}\right)$ is $\mathcal{D}_{\mathbb{Z}^{N}}$-cocompact.

\section{The proof of Theorem 2.3}

We consider the case of real interpolation. The proof for the complex case is completely analogous.

In view of the continuous imbedding $\left(A_{0}, A_{1}\right)_{\theta, q} \subset A_{0}+A_{1}=A_{0}$, it follows that, for each fixed $t$, the operator $M_{t}$ is bounded from $\left(A_{0}, A_{1}\right)_{\theta, q}$ into $A_{1}$. Suppose that $u_{k} \stackrel{\mathcal{D}}{\longrightarrow} 0$ in $\left(A_{0}, A_{1}\right)_{\theta, q}$. Let $\left\{g_{k}\right\}_{k \in \mathbb{N}}$ be an arbitrary sequence in $\mathcal{D}$. Then

$$
g_{k} M_{t} u_{k}=M_{t} h_{g_{k}, t} u_{k}
$$

by property (iii). Since $h_{g_{k}, t} u_{k} \rightarrow 0$ in $\left(A_{0}, A_{1}\right)_{\theta, q}$, we deduce that $M_{t} h_{g_{k}, t} u_{k} \rightarrow 0$ in $A_{1}$ for each fixed $t \in(0,1)$. The cocompactness of the imbedding $A_{1} \subset B_{1}$ and (3.1) now imply that

$$
\lim _{k \rightarrow \infty}\left\|M_{t} u_{k}\right\|_{B_{1}}=0 .
$$

In view of the continuous inclusions $A_{j} \subset B_{j}$ and property (i), we have that $M_{t}$ : $A_{j} \rightarrow B_{j}$ is bounded with

$$
S_{j}:=\sup _{t \in(0,1)}\left\|M_{t}\right\|_{A_{j} \rightarrow B_{j}}<\infty, \quad \text { for } j=0,1 .
$$

Since $M_{t} u_{k} \in B_{0} \cap B_{1}$, we can invoke (6.14) in Appendix A and then (3.3) to obtain that

$$
\begin{aligned}
\left\|M_{t} u_{k}\right\|_{\left(B_{0}, B_{1}\right)_{\theta, q}} & \leq c_{\theta, q}\left\|M_{t} u_{k}\right\|_{B_{0}}^{1-\theta}\left\|M_{t} u_{k}\right\|_{B_{1}}^{\theta} \\
& \leq c_{\theta, q}\left(S_{0}\left\|u_{k}\right\|_{A_{0}}\right)^{1-\theta}\left\|M_{t} u_{k}\right\|_{B_{1}}^{\theta} .
\end{aligned}
$$


Since $\left\{u_{k}\right\}_{k \in \mathbb{N}}$ is necessarily a bounded sequence in the space $\left(A_{0}, A_{1}\right)_{\theta, q}$ (by the Banach-Steinhaus Theorem), and is therefore also bounded in the space $A_{0}$, we can use (3.2) to obtain that

$$
\lim _{k \rightarrow \infty}\left\|M_{t} u_{k}\right\|_{\left(B_{0}, B_{1}\right)_{\theta, q}}=0 .
$$

We now consider the operator $I-M_{t}$ in more detail. By (3.3) we of course have $I-M_{t}: A_{0} \rightarrow B_{0}$ with $\left\|I-M_{t}\right\|_{A_{0} \rightarrow B_{0}} \leq\|I\|_{A_{0} \rightarrow B_{0}}+S_{0}$. Using this estimate, property (ii) and Theorem 6.6 , we obtain that $I-M_{t}$ is a bounded operator from $\left(A_{0}, A_{1}\right)_{\theta, q}$ into $\left(B_{0}, B_{1}\right)_{\theta, q}$ and that

$$
\begin{aligned}
\left\|I-M_{t}\right\|_{\left(A_{0}, A_{1}\right)_{\theta, q} \rightarrow\left(B_{0}, B_{1}\right)_{\theta, q}} & \leq\left\|I-M_{t}\right\|_{A_{0} \rightarrow B_{0}}^{1-\theta}\left\|I-M_{t}\right\|_{A_{1} \rightarrow B_{1}}^{\theta} \\
& \leq\left(\|I\|_{A_{0} \rightarrow B_{0}}+S_{0}\right)^{1-\theta} \sigma(t)^{\theta} .
\end{aligned}
$$

Therefore, with the help of (3.4), we have

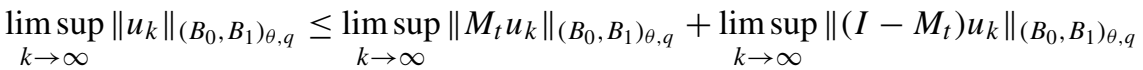

$$
\begin{aligned}
& \leq 0+\limsup _{k \rightarrow \infty}\left\|\left(I-M_{t}\right) u_{k}\right\|_{\left(B_{0}, B_{1}\right)_{\theta, q}} \\
& \leq \limsup _{k \rightarrow \infty}\left(\|I\|_{A_{0} \rightarrow B_{0}}+S_{0}\right)^{1-\theta} \sigma(t)^{\theta}\left\|u_{k}\right\|_{\left(A_{0}, A_{1}\right)_{\theta, q}} .
\end{aligned}
$$

We now use the boundedness of the sequence $\left\{\left\|u_{k}\right\|_{\left(A_{0}, A_{1}\right)_{\theta, q}}\right\}_{k \in \mathbb{N}}$ once more, together with property (ii), to obtain that this last expression is bounded by a quantity which tends to 0 as $t$ tends to 0 . Since we can choose $t$ as small as we please, this shows that $\lim _{k \rightarrow \infty}\left\|u_{k}\right\|_{\left(B_{0}, B_{1}\right)_{\theta, q}}=0$ and completes the proof of the theorem.

\section{Cocompactness of the imbedding $W^{\alpha, p} \subset L^{q}$ for all $\alpha \in(0, \infty)$}

In this section we give the proof of Theorem 2.5.

Let $\Lambda$ be the operator $I-\Delta$ which of course corresponds to the Fourier multiplier $1+|\xi|^{2}$. Note that $\Lambda$ commutes with all of the operators $g \in \mathcal{D}_{\mathbb{Z}^{N}}$, as does each of its powers. Furthermore, $\Lambda^{\gamma / 2}$ defines an isometry between $W^{\alpha+\gamma, p}\left(\mathbb{R}^{N}\right)$

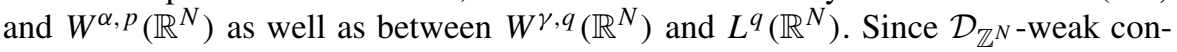
vergence is preserved by each of these isometries and their inverses, we see that

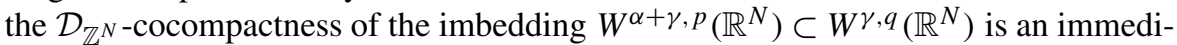

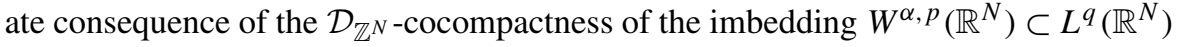
which we will now prove.

We begin by considering the case where $\alpha \in(0,1)$. Here we apply Theorem 2.3 to suitable Banach couples of $L^{p}$ and Sobolev spaces.

We present the first step as the following lemma. Here, as before, $p^{*}=p_{1}^{*}$ is the critical exponent defined in (2.3).

Lemma 4.1 Let $\left(A_{0}, A_{1}\right)=\left(L^{p}\left(\mathbb{R}^{N}\right), W^{1, p}\left(\mathbb{R}^{N}\right)\right)$ and $\left(B_{0}, B_{1}\right)=\left(L^{p}\left(\mathbb{R}^{N}\right)\right.$, $\left.L^{r}\left(\mathbb{R}^{N}\right)\right)$ with $r \in\left(p, p^{*}\right)$. Let $\mathcal{D}=\mathcal{D}_{\mathbb{Z}^{N}}$. 
Let $\rho: \mathbb{R}^{N} \rightarrow[0, \infty)$ be a $C^{\infty}$ function with support contained in the open unit ball $\left\{z \in \mathbb{R}^{N}:|z|<1\right\}$ which satisfies $\int_{\mathbb{R}^{\mathbb{N}}} \rho(x) d x=1$.

Then, for each fixed $t \in(0,1)$ the operator $M_{t}$, which is defined by

$$
\left(M_{t} u\right)(x)=\int_{|z|<1} \rho(z) u(x+t z) d z
$$

is a bounded map of $A_{0}$ into $A_{1}$, and the family $\left\{M_{t}\right\}_{t \in(0,1)}$ satisfies properties (i), (ii) and (iii) of Definition 2.2.

Proof The boundedness of $M_{t}$ from $A_{0}$ into $A_{1}$ for each fixed $t$ is simply the well known mollification property. It is also obvious that $M_{t}: A_{j} \rightarrow A_{j}$ is bounded with $\left\|M_{t}\right\|_{A_{j} \rightarrow A_{j}} \leq 1$ for $j=0,1$ and all $t \in(0,1)$, which gives property (i).

Property (iii) is an immediate consequence of the fact that $\left(M_{t} u\right)(\cdot-y)=$ $M_{t}(u(\cdot-y))$ for each $y \in \mathbb{R}^{N}$. In fact here we can take $h_{g, t}=g$ for each $g \in \mathcal{D}_{\mathbb{Z}^{N}}$ and each $t$.

It remains to prove property (ii). Consider the following identity:

$$
\begin{aligned}
u(x)-M_{t} u(x) & =\int_{|z|<1} \rho(z)[u(x)-u(x+t z)] d z \\
& =-\int_{|z|<1} \rho(z) \int_{0}^{t} z \cdot \nabla u(x+s z) d s d z .
\end{aligned}
$$

Then

$$
\left|u(x)-M_{t} u(x)\right|^{p} \leq \sup _{|y|<1} \rho(y)^{p}\left|\int_{0}^{t} \int_{|z|<1}\right| \nabla u(x+s z)|d z d s|^{p} .
$$

By Hölder's inequality we then have

$$
\left|u(x)-M_{t} u(x)\right|^{p} \leq C t^{p / p^{\prime}} \int_{0}^{t} \int_{|z|<1}|\nabla u(x+s z)|^{p} d z d s .
$$

Integrating with respect to $x$, we obtain

$$
\begin{aligned}
\int_{\mathbb{R}^{N}}\left|u(x)-M_{t} u(x)\right|^{p} d x & \leq C t^{p / p^{\prime}} \int_{0}^{t} \int_{|z|<1} \int_{\mathbb{R}^{N}}|\nabla u(x+s z)|^{p} d x d z d s \\
& =C t^{p / p^{\prime}} \int_{0}^{t} \int_{|z|<1} \int_{\mathbb{R}^{N}}|\nabla u(x)|^{p} d x d z d s \\
& =C t^{1+p / p^{\prime}} \int_{\mathbb{R}^{N}}|\nabla u(x)|^{p} d x .
\end{aligned}
$$

Here, and also later, we will use the following immediate consequence of Hölder's inequality: 
Fact 4.2 The inclusion $L^{p_{0}} \cap L^{p_{1}} \subset L^{p}$ holds whenever $1 \leq p_{0}<p<p_{1} \leq \infty$. Furthermore, the estimate

$$
\|f\|_{p} \leq\|f\|_{p_{0}}^{1-\theta}\|f\|_{p_{1}}^{\theta}
$$

holds for each $f \in L^{p_{0}} \cap L^{p_{1}}$, where $\theta=\Theta\left(p_{0}, p, p_{1}\right):=\frac{\frac{1}{p_{0}}-\frac{1}{p}}{\frac{1}{p_{0}}-\frac{1}{p_{1}}} \in(0,1)$.

Let $s$ be some number satisfying $r<s<p^{*}$. Then $p<r<s$ and so Fact 4.2 gives us that

$$
\left\|u-M_{t} u\right\|_{r} \leq\left\|u-M_{t} u\right\|_{p}^{1-\theta}\left\|u-M_{t} u\right\|_{s}^{\theta}, \quad \text { where } \theta=\frac{\frac{1}{p}-\frac{1}{r}}{\frac{1}{p}-\frac{1}{s}} \in(0,1) .
$$

We estimate $\left\|u-M_{t} u\right\|_{p}$ and $\left\|u-M_{t} u\right\|_{s}$ using, respectively, (4.2) and the Sobolev imbedding theorem. Substituting these estimates in (4.4), and noting that $1+p / p^{\prime}=p$, we obtain that

$$
\begin{aligned}
\left\|u-M_{t} u\right\|_{r} & \leq C\left(t^{p}\|u\|_{W^{1, p}}\right)^{1-\theta}\left(\left\|u-M_{t} u\right\|_{W^{1, p}}\right)^{\theta} \\
& \leq C t^{(1-\theta) p}\|u\|_{W^{1, p}} .
\end{aligned}
$$

This establishes property (ii) and completes the proof of the lemma.

We will now prove the assertion of Theorem 2.5 for $\alpha \in(0,1)$ and for some particular value of $q \in\left(p, p_{\alpha}^{*}\right)$.

For the number $p \in(1, \infty)$ appearing in the statement of Theorem 2.5, and for some number $r$ in $\left(p, p^{*}\right)$ we let $\left(A_{0}, A_{1}\right)$ and $\left(B_{0}, B_{1}\right)$ be the same couples $\left(L^{p}\left(\mathbb{R}^{N}\right), W^{1, p}\left(\mathbb{R}^{N}\right)\right)$ and $\left(L^{p}\left(\mathbb{R}^{N}\right), L^{r}\left(\mathbb{R}^{N}\right)\right)$ which appear in Lemma 4.1. Let us also choose the group $\mathcal{D}$ and the family of operators $\left\{M_{t}\right\}_{t \in(0,1)}$ to be as in Lemma 4.1.

We know, using Theorem 2.4, that $A_{1}$ is $\mathcal{D}_{\mathbb{Z}^{N}}$-cocompactly imbedded in $B_{1}$. This, together with Lemma 4.1, provides us with all the conditions required for applying Theorem 2.3 in this context. More specifically, if we invoke the statement about complex interpolation spaces in Theorem 2.3, we obtain that $\left[L^{p}\left(\mathbb{R}^{N}\right), W^{1, p}\left(\mathbb{R}^{N}\right)\right]_{\theta}$ is

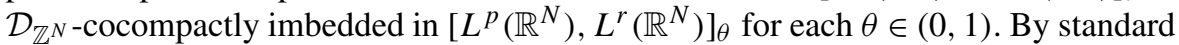
results (see Appendix A), these two spaces are $W^{\theta, p}\left(\mathbb{R}^{N}\right)$ and $L^{s_{0}}\left(\mathbb{R}^{N}\right)$ respectively, where $s_{0}$ is the number in the interval $(p, r)$ given by

$$
\frac{1}{s_{0}}=\frac{1-\theta}{p}+\frac{\theta}{r} \text {. }
$$

Setting $\theta=\alpha$, we see that this establishes our result for $q=s_{0}$. It will now be easy to extend the proof to all $q \in\left(p, p_{\alpha}^{*}\right)$ :

Let $\left\{u_{k}\right\}_{k \in \mathbb{N}}$ be an arbitrary sequence in $W^{\alpha, p}$ which converges $\mathcal{D}_{\mathbb{Z}^{N}}$-weakly to 0 .

Given an arbitrary $q$ in $\left(p, p_{\alpha}^{*}\right)$ we choose $r \in\left(p, p^{*}\right)$ sufficiently close to $p$ so that the number $s_{0}$ given by (4.5), with $\theta=\alpha$, satisfies $p<s_{0}<q$. By the previous step of our argument we also have that $\lim _{k \rightarrow \infty}\left\|u_{k}\right\|_{L^{s_{0}\left(\mathbb{R}^{N}\right)}}=0$. Now let us 
choose some number $s_{1} \in\left(q, p_{\alpha}^{*}\right)$. By the Sobolev imbedding theorem, the sequence $\left\{u_{k}\right\}_{k \in \mathbb{N}}$, which is bounded in $W^{1, p}\left(\mathbb{R}^{N}\right)$, must also be bounded in $L^{s_{1}}\left(\mathbb{R}^{N}\right)$. Finally, we use Fact 4.2 to bound $\left\|u_{k}\right\|_{q}$ by $\left\|u_{k}\right\|_{s_{0}}^{1-\beta}\left\|u_{k}\right\|_{s_{1}}^{\beta}$ for a suitable number $\beta \in(0,1)$. This suffices to complete the proof of Theorem 2.5 for the case $\alpha \in(0,1)$.

The case where $\alpha=1$ is of course Theorem 2.4. So it remains to deal with the easy case where $\alpha>1$.

Let $p$ and $q$ be as in the statement of the theorem. Noting that we always have $p<p^{*}$, let us choose numbers $q_{0}$ and $q_{1}$ which satisfy $p<q_{0}<\min \left\{p^{*}, q\right\}$ and $q<q_{1}<p_{\alpha}^{*}$. Consider an arbitrary sequence $\left\{u_{k}\right\}_{k \in \mathbb{N}}$ in $W^{\alpha, p}\left(\mathbb{R}^{N}\right)$ which is $\mathcal{D}_{\mathbb{Z}^{N-}}$ weakly convergent to zero. Since in this case $W^{\alpha, p}\left(\mathbb{R}^{N}\right)$ is continuously imbedded into $W^{1, p}\left(\mathbb{R}^{N}\right)$, we have that $u_{k}\left(\cdot-y_{k}\right) \rightarrow 0$ in $W^{1, p}\left(\mathbb{R}^{N}\right)$ for any sequence $\left\{y_{k}\right\}_{k \in \mathbb{N}}$ of elements of $\mathbb{Z}^{N}$, i.e., $u_{k}$ is $\mathcal{D}_{\mathbb{Z}^{N}}$-weakly convergent in $W^{1, p}\left(\mathbb{R}^{N}\right)$. Then, by Theorem 2.4, $\lim _{k \rightarrow \infty}\left\|u_{k}\right\|_{q_{0}}=0$.

Since $q_{0}<q<q_{1}$, Fact 4.2 gives us that

$$
\left\|u_{k}\right\|_{q} \leq\left\|u_{k}\right\|_{q_{0}}^{1-\theta}\left\|u_{k}\right\|_{q_{1}}^{\theta}, \quad \text { where } \theta=\frac{\frac{1}{q_{0}}-\frac{1}{q}}{\frac{1}{q_{0}}-\frac{1}{q_{1}}} \in(0,1) .
$$

Then, since $W^{\alpha, p}\left(\mathbb{R}^{N}\right)$ is continuously imbedded into $L^{q_{1}}\left(\mathbb{R}^{N}\right)$, we have $\left\|u_{k}\right\|_{q} \leq$ $C\left\|u_{k}\right\|_{q_{0}}^{1-\theta}\left\|u_{k}\right\|_{W^{\alpha, p}}^{\theta}$. Since (again by the Banach-Steinhaus Theorem) weakly convergent sequences are bounded, we obtain that $\left\|u_{k}\right\|_{q} \leq C\left\|u_{k}\right\|_{q_{0}}^{\theta} \rightarrow 0$.

\section{Cocompact imbeddings of Besov spaces and the proofs of Theorems 2.6 and 2.8}

The following lemma will be the main component of the proof of Theorem 2.6.

Lemma 5.1 Suppose that $m_{0}, m_{1} \in \mathbb{R}, 0 \leq m_{1}<m_{0}, 1<p_{0}<p_{1}<\infty$, and assume further that

$$
\frac{1}{p_{0}}-\frac{1}{p_{1}}<\frac{m_{0}-m_{1}}{N}
$$

For each $t \in(0,1)$, the operator $M_{t}$ defined by (4.1) is a bounded map from $W^{m_{0}, p_{0}}\left(\mathbb{R}^{N}\right)$ to $W^{m_{1}, p_{1}}\left(\mathbb{R}^{N}\right)$ and satisfies

$$
\lim _{t \rightarrow 0}\left\|I-M_{t}\right\|_{W^{m_{0}, p_{0}}\left(\mathbb{R}^{N}\right) \rightarrow W^{m_{1}, p_{1}}\left(\mathbb{R}^{N}\right)}=0 .
$$

Proof We begin by observing that the conditions on $p_{0}$ and $p_{1}$ in the statement of the lemma are equivalent to

$$
1<p_{0}<p_{1}<\left(p_{0}\right)_{m_{0}-m_{1}}^{*}
$$

(The notation here is as specified in (2.3), and this equivalence holds whether or not $\left(p_{0}\right)_{m_{0}}^{*}$ is finite.) 
We shall make use once more of the operator $\Lambda=I-\Delta$ which was introduced at the beginning of Sect. 4, noting that $\Lambda$ and each of its powers all commute with all of the operators $M_{t}$. Since $\Lambda^{m_{1} / 2}$ defines an isometry between $W^{m_{1}, p_{1}}\left(\mathbb{R}^{N}\right)$ and $L^{p_{1}}\left(\mathbb{R}^{N}\right)$ as well as between $W^{m_{0}, p_{0}}\left(\mathbb{R}^{N}\right)$ and $W^{m_{0}-m_{1}, p_{0}}\left(\mathbb{R}^{N}\right)$, it suffices to prove the lemma in the case where the two parameters $m_{0}$ and $m_{1}$ are replaced by $m_{0}^{\prime}=$ $m_{0}-m_{1}$ and $m_{1}^{\prime}=m_{1}-m_{1}=0$, i.e. we can suppose that $m_{1}=0$. Note that this "shift" of the values of $m_{0}$ and $m_{1}$ does not change the stated conditions on $p_{0}$ and $p_{1}$.

Case 1: Assume first that $m_{0} \geq 1$. By Lemma 4.1, we have

$$
\lim _{t \rightarrow 0}\left\|I-M_{t}\right\|_{W^{1, p_{0} \rightarrow L^{r}}}=0 \quad \text { for each } r \in\left(p_{0},\left(p_{0}\right)^{*}\right) .
$$

This also implies that

$$
\lim _{t \rightarrow 0}\left\|I-M_{t}\right\|_{W^{m_{0}}, p_{0} \rightarrow L^{r}}=0 .
$$

Sub-case 1.1: If $p_{1}=r$ the lemma is proved.

Sub-case 1.2: If $p_{1}>r$ then we can obtain (5.2) by using Fact 4.2 with $r, p_{1}$ and some number $s \in\left(p_{1},\left(p_{0}\right)_{m_{0}}^{*}\right)$ now assuming the roles of $p_{0}, p$ and $p_{1}$ respectively. More precisely, for each $f \in W^{m_{0}, p_{0}}$ and for $\theta=\Theta\left(r, p_{1}, s\right) \in(0,1)$, we have

$$
\begin{aligned}
\left\|\left(I-M_{t}\right) f\right\|_{L^{p_{1}}} & \leq\left\|\left(I-M_{t}\right) f\right\|_{L^{r}}^{1-\theta}\left\|\left(I-M_{t}\right) f\right\|_{L^{s}}^{\theta} \\
& \leq\left(\left\|I-M_{t}\right\|_{W^{m_{0}, p_{0}} \rightarrow L^{r}}\|f\|_{W^{m_{0}, p_{0}}}\right)^{1-\theta}\left(2\|f\|_{L^{s}}\right)^{\theta} .
\end{aligned}
$$

Since $p_{0}<s<\left(p_{0}\right)_{m_{0}}^{*}$ we have that $\|f\|_{L^{s}}$ is bounded by a constant multiple of $\|f\|_{W^{m_{0}}, p_{0}}$ which we can substitute in (5.7) and then use (5.5) to obtain the required property (5.2) in this case.

Sub-case 1.3: If $p_{1}<r$, we use an argument similar to the one for Sub-case 1.2. This time we apply Fact 4.2 with $p_{0}, p_{1}$ and $r$ in the roles of $p_{0}, p$ and $p_{1}$. Accordingly, analogously to (5.10), for each $f \in W^{m_{0}, p_{0}}$ and for $\theta=\Theta\left(p_{0}, p_{1}, r\right) \in(0,1)$, we have that

$$
\begin{aligned}
\left\|\left(I-M_{t}\right) f\right\|_{L^{p_{1}}} & \leq\left\|\left(I-M_{t}\right) f\right\|_{L^{p_{0}}}^{1-\theta}\left\|\left(I-M_{t}\right) f\right\|_{L^{r}}^{\theta} \\
& \leq\left(2\|f\|_{L^{p_{0}}}\right)^{1-\theta}\left(\left\|I-M_{t}\right\|_{W^{m_{0}, p_{0}} \rightarrow L^{r}}\|f\|_{W^{m_{0}, p_{0}}}\right)^{\theta} .
\end{aligned}
$$

Obviously $\|f\|_{L^{p_{0}}} \leq\|f\|_{W^{m_{0}, p_{0}}}$ and so the proof is also complete in this case.

Case 2: If $0<m_{0}<1$, then we apply Theorem 6.6 to the operator $T=I-M_{t}$ and the couples $\left(A_{0}, A_{1}\right)=\left(L^{p_{0}}, W^{1, p_{0}}\right)$ and $\left(B_{0}, B_{1}\right)=\left(L^{p_{0}}, L^{r}\right)$ where $r \in$ $\left(p_{0},\left(p_{0}\right)^{*}\right)$. We choose $\theta=m_{0}$ and use the facts (see Appendix A) that $W^{m_{0}, p_{0}}=$ $\left[L^{p_{0}}, W^{1, p_{0}}\right]_{m_{0}}$ and $\left[L^{p_{0}}, L^{r}\right]_{m_{0}}=L^{s_{0}}$, where

$$
\frac{1}{s_{0}}=\frac{1-m_{0}}{p_{0}}+\frac{m_{0}}{r} .
$$

Thus we obtain that

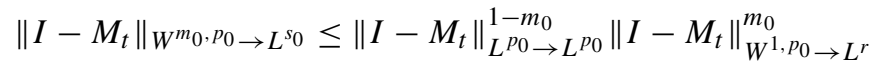




$$
\leq 2^{1-m_{0}}\left\|I-M_{t}\right\|_{W^{1, p_{0} \rightarrow L^{r}}}^{m_{0}} .
$$

Since we are free to choose $r$ arbitrarily close to $p_{0}$, we see from (5.8) that we can also have $s_{0}$ arbitrarily close to $p_{0}$. So, keeping (5.3) in mind, let us choose $r$ so that $s_{0}<p_{1}$ and let us choose a second number $s_{1} \in\left(p_{1},\left(p_{0}\right)_{m_{0}}^{*}\right)$. Now we use Fact 4.2 once more: For each $f \in W^{m_{0}, p_{0}}$, and for $\theta=\Theta\left(s_{0}, p_{1}, s_{1}\right) \in(0,1)$, we have

$$
\begin{aligned}
& \left\|\left(I-M_{t}\right) f\right\|_{L^{p_{1}}} \leq\left\|\left(I-M_{t}\right) f\right\|_{L^{s_{0}}}^{1-\theta}\left\|\left(I-M_{t}\right) f\right\|_{L^{s_{1}}}^{\theta} \\
& \leq\left(\left\|I-M_{t}\right\|_{W^{m_{0}}, p_{0} \rightarrow L^{s_{0}}}\|f\|_{W^{m_{0}}, p_{0}}\right)^{1-\theta}\left(2\|f\|_{L^{s_{1}}}\right)^{\theta} .
\end{aligned}
$$

The fact that $s_{1} \in\left(p_{0},\left(p_{0}\right)_{m_{0}}^{*}\right)$ ensures that $\|f\|_{L^{s_{1}}}$ is bounded by a constant multiple of $\|f\|_{W^{m_{0}}, p_{0}}$. After we substitute this in (5.10) and apply (5.9) and then (5.4), we obtain (5.2) in this final case, and so complete the proof of the lemma.

After these preparations, the proof of Theorem 2.6 is almost immediate. Let $\epsilon \epsilon$ $(0, \beta / 2)$ and let $\alpha_{0}=\alpha+\epsilon, \alpha_{1}=\alpha-\epsilon, \beta_{0}=\beta+\epsilon$ and $\beta_{1}=\beta-\epsilon$. Consider the Banach couples

$$
\begin{aligned}
& \left(A_{0}, A_{1}\right)=\left(W^{\alpha_{0}, p_{0}}\left(\mathbb{R}^{N}\right), W^{\alpha_{1}, p_{0}}\left(\mathbb{R}^{N}\right)\right) \quad \text { and } \\
& \left(B_{0}, B_{1}\right)=\left(W^{\beta_{0}, p_{1}}\left(\mathbb{R}^{N}\right), W^{\beta_{1}, p_{1}}\left(\mathbb{R}^{N}\right)\right) .
\end{aligned}
$$

Let $\lambda=\frac{N}{p_{0}}-\frac{N}{p_{1}}$. For $j=0,1$, since $\alpha_{j}-\beta_{j}=\alpha-\beta>\lambda$, we obtain from

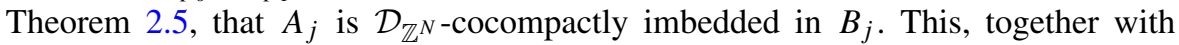
Lemma 5.1, shows that the conditions for applying Theorem 2.3 are fulfilled. So we can deduce that $\left(A_{0}, A_{1}\right)_{\theta, q}$ is $\mathcal{D}_{\mathbb{Z}^{N}}$-cocompactly imbedded into $\left(B_{0}, B_{1}\right)_{\theta, q}$ for each $\theta \in(0,1)$ and $q \in[1, \infty]$. In particular, if we choose $\theta=1 / 2$ we obtain the assertion of the theorem.

We now turn to the proof of Theorem 2.8. Obviously in view of (6.17), (6.8) and Proposition 1.4, it suffices to consider the case where $q_{0}=q$. Fix some $\theta \in(0,1)$ and define $s_{0}$ and $r$ so that they satisfy $s=\theta s_{0}$ and

$$
\frac{1}{q}=\frac{1-\theta}{p}+\frac{\theta}{r} .
$$

We next want to show that

$$
q<r<p_{s_{0}}^{*} .
$$

The first inequality of (5.12) follows from (5.11) and the fact that $p<q$. The second inequality of (5.12) is equivalent to

$$
\frac{1}{r}>\frac{1}{p}-\frac{s_{0}}{N}
$$

which readily follows from $1 / q>1 / p-s / N=1 / p-\theta s_{0} / N$ and (5.11). 


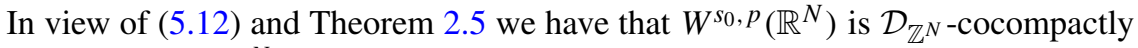
imbedded into $L^{r}\left(\mathbb{R}^{N}\right)$. Then, by Theorem 2.3 it follows that the imbedding

$$
\left(L^{p}, W^{s_{0}, p}\right)_{\theta, r} \subset\left(L^{p}, L^{r_{0}}\right)_{\theta, r}
$$

is $\mathcal{D}_{\mathbb{Z}^{N}}$-cocompact. Using (6.17) and (6.15), we identify the above imbedding as $B^{s, p, r} \subset L^{r}$.

\section{Compactness and existence of minimizers}

\subsection{Compact imbeddings of radial subspaces}

There are many known examples where a function space $A$ is cocompactly imbedded into some other function space $B$, and some significant subspace $\widetilde{A}$ of $A$ is compactly imbedded into the same space $B$.

For example, in the case where $A=W^{m, p}\left(\mathbb{R}^{N}\right)$ and $\widetilde{A}$ is its subspace of functions supported in some fixed compact subset of $\mathbb{R}^{N}$, then the usual subcritical Sobolev imbedding of $A$ is cocompact, and that of $\widetilde{A}$ is compact (by the Rellich-Kondrashov lemma). In this subsection we consider a different case, where $\widetilde{A}$ is the subspace of all radial functions in some function space $A$.

We refer to [34] and also to Chaps. 3 and 4 of [40] for more detailed discussions of these kind of phenomena.

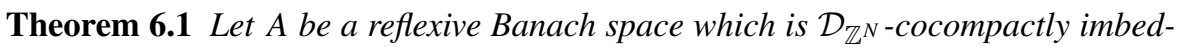
ded into $L^{p}\left(\mathbb{R}^{N}\right)$ for some $p>1$. Suppose that every weakly convergent sequence in A has a subsequence which converges a.e. Suppose furthermore that $A$ is $\mathbb{R}^{N}$-shift invariant and also rotation invariant, i.e. that

$$
\|u \circ \omega\|_{A}=\|u\|_{A}
$$

for each $u \in A$ and for each $\omega: \mathbb{R}^{N} \rightarrow \mathbb{R}^{N}$ which is either a shift by some element of $\mathbb{R}^{N}$ or an element of $O(N)$. Let $A_{R}$ denote the subspace of radially symmetric functions in $A$.

Then the imbedding of $A_{R}$ into $L^{p}\left(\mathbb{R}^{N}\right)$ is compact.

Proof Let $\left\{u_{k}\right\}_{k \in \mathbb{N}}$ be an arbitrary bounded sequence in $A_{R}$. We have to show that it has a subsequence which converges in norm in $L^{p}\left(\mathbb{R}^{N}\right)$. Thus, by passing if necessary to a subsequence, we may assume without loss of generality that $u_{k}$ converges weakly in $A$ to some limit which, again without loss of generality, may be assumed to be 0 . This immediately implies that, for any finite subset $E$ of $\mathbb{Z}^{N}$ and any sequence $\left\{y_{k}\right\}_{k \in \mathbb{N}}$ in $E$, the sequence $u_{k}\left(\cdot-y_{k}\right)$ tends to 0 weakly in $A$. If the sequence $\left\{u_{k}\right\}_{k \in \mathbb{N}}$ is $\mathcal{D}_{\mathbb{Z}^{N}}$-weakly convergent to 0 in $A$ then, in view of the $\mathcal{D}_{\mathbb{Z}^{N}}$-cocompactness of the embedding of $A$ into $L^{p}\left(\mathbb{R}^{N}\right)$, the proof is complete. Therefore we shall assume the existence of a sequence $\left\{y_{k}\right\}_{k \in \mathbb{N}}$ in $\mathbb{R}^{N}$ for which $u_{k}\left(\cdot-y_{k}\right)$ does not converge weakly to 0 and show that this leads to a contradiction. After making this assumption, further 
passages to subsequences, if necessary, enable us to assume that $u_{k}\left(\cdot-y_{k}\right)$ does have a non zero weak limit $w \in A$ and also that

$$
\lim _{k \rightarrow \infty} \frac{y_{k}}{\left|y_{k}\right|}=z_{*}
$$

for some point $z_{*}$ on the unit sphere of $\mathbb{R}^{N}$. The first of these properties tells us that the sequence $\left\{y_{k}\right\}_{k \in \mathbb{N}}$ cannot be confined to any finite subset $E$ of $\mathbb{Z}^{N}$. So we may also assume that $\left|y_{k}\right| \rightarrow \infty$.

Since $u_{k} \circ \omega=u_{k}$, for every $\omega \in O(N)$, it follows that $u_{k}\left(\cdot-\omega^{-1} y_{k}\right) \rightarrow w \circ \omega$. Let $\left\{\omega_{n}\right\}_{n \in \mathbb{N}}$ be a sequence of elements of $O(N)$ which satisfy $\omega_{i}^{-1} z_{*} \neq \omega_{j}^{-1} z_{*}$ whenever $i \neq j$. Then it readily follows from (6.1) that $\left|\omega_{i}^{-1} y_{k}-\omega_{j}^{-1} y_{k}\right| \rightarrow \infty$ whenever $i \neq j$. Taking into account the continuity of the imbedding into $L^{p}$ and the assumption about a.e. convergence, passing yet again if necessary to a subsequence, and then applying the iterated Brezis-Lieb lemma (Proposition 6.7) with $y_{k}^{(n)}=-\omega_{n}^{-1} y_{k}$ for each $k$ and $n$, we conclude from (6.20) that, for any $M \in \mathbb{N}$,

$$
\infty>\liminf _{k \rightarrow \infty} \int_{\mathbb{R}^{N}}\left|u_{k}\right|^{p} \geq \sum_{i=1}^{M} \int_{\mathbb{R}^{N}}\left|w \circ \omega_{i}\right|^{p}=M \int_{\mathbb{R}^{N}}|w|^{p} .
$$

Since $M$ is an arbitrary integer, we obtain the contradiction required to complete the proof of the theorem.

Theorem 6.1 can be immediately combined with Theorem 2.5 to give the following:

Corollary 6.2 Let $\alpha>0, p \in(1, \infty), N<p \alpha$ and $q \in\left(p, p_{\alpha}^{*}\right)$. Then the imbedding of $W_{R}^{\alpha, p}\left(\mathbb{R}^{N}\right)$ into $L^{q}\left(\mathbb{R}^{N}\right)$ is compact.

This is of course a known result, see Strauss [35].

Similarly, combining 6.1 with Theorem 2.8 will give us:

Corollary 6.3 Let $s>0,1<p<\infty, p \leq q_{0}<q<p_{s}^{*}$. Then the subspace $B_{R}^{s, p, q_{0}}\left(\mathbb{R}^{N}\right)$ of radially symmetric functions in $B^{s, p, q_{0}}\left(\mathbb{R}^{N}\right)$ is compactly imbedded into $L^{q}\left(\mathbb{R}^{N}\right)$.

This result may be compared to the compactness of imbeddings of radial subspaces of Besov spaces obtained by Sickel and Skrzypczak [32]. We remark also that some results of this kind may be obtained by interpolation of imbeddings of radial subspaces of classical Sobolev spaces.

\subsection{Existence of minimizers}

For $p=2$ the (fractional) Sobolev space $W^{\alpha, 2}\left(\mathbb{R}^{N}\right)$ is of course a Hilbert space, which is customarily denoted by $H^{\alpha}$. One of its natural equivalent norms is given by

$$
\|f\|_{H^{\alpha}}=\left(\int_{\mathbb{R}^{N}}\left(1+|\xi|^{2}\right)^{\alpha}|\widehat{f}(\xi)|^{2} d \xi\right)^{1 / 2} .
$$


The following two theorems hold for the norm (6.2) and also for any other equivalent Hilbert norm on $H^{\alpha}$ which, like (6.2), is invariant under lattice translations. When $\alpha$ is a positive integer these theorems are well known, the first being due to Berezstycki-P.-L. Lions [4] and the second to P.-L. Lions [22]. Later versions of the proofs of their two results can be found, for example in Struwe [36]. Our extensions here to the case where $\alpha$ is not an integer are straightforward adaptations of the standard proofs.

Theorem 6.4 For each $\alpha>0$ and each $q \in\left(2,2_{\alpha}^{*}\right)$, the infinimum

$$
\kappa:=\inf _{\|u\|_{L^{q}\left(\mathbb{R}^{N}\right)}=1}\|u\|_{H^{\alpha}\left(\mathbb{R}^{N}\right)}^{2}
$$

is attained.

Proof Let $\left\{u_{k}\right\}_{k \in \mathbb{N}}$ be a minimizing sequence, that is, $\lim _{k \rightarrow \infty}\left\|u_{k}\right\|_{H^{\alpha}\left(\mathbb{R}^{N}\right)}^{2}=\kappa$ and $\left\|u_{k}\right\|_{L^{q}\left(\mathbb{R}^{N}\right)}=1$. Suppose that, for every sequence $\left\{y_{k}\right\}_{k \in \mathbb{N}}$ in $\mathbb{Z}^{\mathbb{N}}$, the sequence $\left\{u_{k}\left(\cdot-y_{k}\right)\right\}_{k \in \mathbb{N}}$ converges weakly in $H^{\alpha}$ to 0 . Then $u_{k} \rightarrow 0$ in $L^{q}\left(\mathbb{R}^{N}\right)$, since

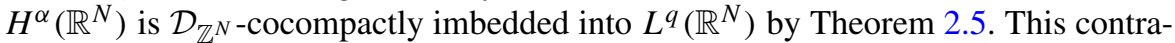
dicts the assumption $\left\|u_{k}\right\|_{L^{q}\left(\mathbb{R}^{N}\right)}=1$. Consequently, there exist a (possibly renamed) subsequence $\left\{y_{k}\right\}_{k \in \mathbb{N}}$ and a function $w \in H^{\alpha}\left(\mathbb{R}^{N}\right) \backslash\{0\}$, such that $u_{k}\left(\cdot-y_{k}\right)$ converges to $w$ weakly in $H^{\alpha}$ and also pointwise a.e. (Here we take into account that weak convergence in $H^{\alpha}$ implies convergence locally in measure.) Furthermore, the sequence $v_{k}=\left\{u_{k}\left(\cdot-y_{k}\right)\right\}_{k \in \mathbb{N}}$ is also a minimizing sequence. Then

$$
\begin{aligned}
\kappa & =\left\|v_{k}\right\|_{H^{\alpha}\left(\mathbb{R}^{N}\right)}^{2}+o(1)=\left\|\left(v_{k}-w\right)+w\right\|_{H^{\alpha}\left(\mathbb{R}^{N}\right)}^{2}+o(1) \\
& =\left\|v_{k}-w\right\|_{H^{\alpha}\left(\mathbb{R}^{N}\right)}^{2}+\|w\|_{H^{\alpha}\left(\mathbb{R}^{N}\right)}^{2}+2\left\langle v_{k}-w, w\right\rangle+o(1) \\
& =\left\|v_{k}-w\right\|_{H^{\alpha}\left(\mathbb{R}^{N}\right)}^{2}+\|w\|_{H^{\alpha}\left(\mathbb{R}^{N}\right)}^{2}+o(1) .
\end{aligned}
$$

By the Brezis-Lieb lemma,

$$
1=\left\|v_{k}\right\|_{L^{q}\left(\mathbb{R}^{N}\right)}^{q}=\left\|v_{k}-w\right\|_{L^{q}\left(\mathbb{R}^{N}\right)}^{q}+\|w\|_{L^{q}\left(\mathbb{R}^{N}\right)}^{q}+o(1) .
$$

Since $\|f\|_{H^{\alpha}}^{2} \geq \kappa\|f\|_{L^{q}}^{2}$ for each $f \in H^{\alpha}$, we can deduce from (6.4) that

$$
\kappa \geq \kappa\left\|v_{k}-w\right\|_{L^{q}\left(\mathbb{R}^{N}\right)}^{2}+\kappa\|w\|_{L^{q}\left(\mathbb{R}^{N}\right)}^{2}+o(1) .
$$

This in turn, in view of (6.5), implies that

$$
\kappa \geq \kappa\left(1-\|w\|_{L^{q}\left(\mathbb{R}^{N}\right)}^{q}\right)^{2 / q}+\kappa\|w\|_{L^{q}\left(\mathbb{R}^{N}\right)}^{2} .
$$

Since $q>2$ and $w \neq 0$, the last inequality holds true only if $\|w\|_{L^{q}\left(\mathbb{R}^{N}\right)}=1$. The weak lower semicontinuity of the norm implies that $\|w\|_{H^{\alpha}\left(\mathbb{R}^{N}\right)}^{2} \leq \kappa$. But then $\|w\|_{H^{\alpha}\left(\mathbb{R}^{N}\right)}^{2}=\kappa$, since $\kappa$ is the infimum value for such expressions. Therefore, $v_{k}$ converges in the norm of $H^{\alpha}\left(\mathbb{R}^{N}\right)$ to a minimum element $w$. 
Our second theorem deduces the existence of a minimizer as a consequence of a penalty condition.

Theorem 6.5 Assume that the function $b \in C\left(\mathbb{R}^{N}\right)$ has a limit at infinity and that $0<b_{\infty}:=\lim _{|x| \rightarrow \infty} b(x)<b(x)$ for all $x \in \mathbb{R}^{N}$. Then, for each $\alpha>0$ and each $q \in\left(2,2_{\alpha}^{*}\right)$, the infimum

$$
\tilde{\kappa}:=\inf _{\int_{\mathbb{R}^{N}} b(x)|u|^{q} d x=1}\|u\|_{H^{\alpha}\left(\mathbb{R}^{N}\right)}
$$

is attained.

Proof Let $F(u):=\int_{\mathbb{R}^{N}} b(x)|u|^{p} d x, \quad F_{0}(u):=\int_{\mathbb{R}^{N}} b_{\infty}|u|^{p} d x$ and $\Psi(u):=$ $\int_{\mathbb{R}^{N}}\left(b(x)-b_{\infty}\right)|u|^{p} d x$. Note that $\Psi(u)>0$ unless $u=0$. It is easy to show that $\Psi$ is weakly continuous in $H^{\alpha}\left(\mathbb{R}^{N}\right)$, by fixing an $\epsilon>0$ and dividing the domain of integration into $\left\{b(x)-b_{\infty} \leq \epsilon\right\}$ and the bounded region $\left\{b(x)-b_{\infty}>\epsilon\right\}$. Let $\left\{u_{k}\right\}_{k \in \mathbb{N}}$ be a minimizing sequence, that is, $\lim _{k \rightarrow \infty}\left\|u_{k}\right\|_{H^{\alpha}\left(\mathbb{R}^{N}\right)}^{2}=\kappa$ and $F\left(u_{k}\right)=1$. Without loss of generality, we may assume that $u_{k} \rightarrow w$ in $H^{\alpha}\left(\mathbb{R}^{N}\right)$. As in the proof of Theorem 6.4,

$$
\tilde{\kappa}=\left\|u_{k}\right\|_{H^{\alpha}\left(\mathbb{R}^{N}\right)}^{2}+o(1)=\left\|u_{k}-w\right\|_{H^{\alpha}\left(\mathbb{R}^{N}\right)}^{2}+\|u\|_{H^{\alpha}\left(\mathbb{R}^{N}\right)}^{2}+o(1) .
$$

So, if we write $F(u)$ as a sum of $F_{0}(u)=\|u\|_{L^{q}\left(\mathbb{R}^{N}\right)}^{q}$ and the weakly-continuous functional $\Psi(u)$, the Brezis-Lieb lemma applied to $F_{0}$ gives us that

$$
1=F\left(u_{k}\right)=\lim _{k \rightarrow \infty} F_{0}\left(u_{k}-w\right)+F_{0}(w)+\Psi(w) \leq \lim _{k \rightarrow \infty} F\left(u_{k}-w\right)+F(w)
$$

where the inequality is strict unless $u_{k} \rightarrow w$ in $L^{q}\left(\mathbb{R}^{N}\right)$. Comparing (6.6) and (6.7), we obtain

$\tilde{\kappa} \geq \tilde{\kappa}\left\|u_{k}-w\right\|_{L^{q}\left(\mathbb{R}^{N}\right)}^{2}+\tilde{\kappa}\|w\|_{L^{q}\left(\mathbb{R}^{N}\right)}^{2}+o(1) \geq \tilde{\kappa}(1-F(w))^{2 / q}+\tilde{\kappa} F(w)^{2 / q}+o(1)$.

Since $q>2$, the last inequality holds true only if $F(w)=1$ or $w=0$. If, however, $w=0$, by the weak continuity of $\Psi$ and the Brezis-Lieb lemma we have $F\left(u_{k}\right)=F_{0}\left(u_{k}\right)$, which implies that $\tilde{\kappa} \geq \kappa$. On the other hand, substitution of the (renormalized) minimizer for 6.3 yields $\tilde{\kappa}<\kappa$, a contradiction. Consequently, $F(w)=1$ and one can verify that $w$ is a minimizer by a literal repetition of the last steps of the proof of Theorem 6.4.

Acknowledgements We thank the referees for some very helpful comments, in particular with regard to Theorem 6.1.

Open Access This article is distributed under the terms of the Creative Commons Attribution Noncommercial License which permits any noncommercial use, distribution, and reproduction in any medium, provided the original author(s) and source are credited. 


\section{Appendix A: Basics of interpolation theory and Besov spaces}

We summarize here the basic definitions and facts about interpolation spaces generated by the "real method" (J.-L. Lions-J. Peetre [21]) and by the "complex method" (A.P. Calderón [9]). For more details one can refer, e.g., to [1, 3, 5, 7] and/or [41].

Banach couples Suppose that $A_{0}$ and $A_{1}$ are Banach spaces which are both linear subspaces of some Hausdorff linear topological space $\mathcal{A}$, and the identity maps from $A_{0}$ into $\mathcal{A}$ and from $A_{1}$ into $\mathcal{A}$ are both continuous. Then we say that $\left(A_{0}, A_{1}\right)$ is a Banach couple. (It is not difficult to see that this definition is equivalent to the seemingly more stringent definition where $\mathcal{A}$ is also required to be a Banach space.)

For each Banach couple $\left(A_{0}, A_{1}\right)$ it is clear that the space $A_{0}+A_{1}$ normed by $\|a\|_{A_{0}+A_{1}}:=\inf \left\{\left\|a_{0}\right\|_{A_{0}}+\left\|a_{1}\right\|_{A_{1}}: a_{0} \in A_{0}, a_{1} \in A_{1}, a=a_{0}+a_{1}\right\}$ is also a Banach space.

The real interpolation method (J.-L. Lions-J. Peetre [21]) There are several equivalent definitions of the real method interpolation spaces $\left(A_{0}, A_{1}\right)_{\theta, q}$ of Lions-Peetre, and here we give one of them that uses the Peetre $K$-functional. This is the functional defined for each fixed $t>0$ and each $a \in A_{0}+A_{1}$, by

$$
K\left(t, a ; A_{0}, A_{1}\right):=\inf \left\{\left\|a_{0}\right\|_{A_{0}}+t\left\|a_{1}\right\|_{A_{1}}: a_{0} \in A_{0}, a_{1} \in A_{1}, a=a_{0}+a_{1}\right\} .
$$

Obviously, $\left\{K\left(t, \cdot ; A_{0}, A_{1}\right)\right\}_{t>0}$ is a family of equivalent norms on $A_{0}+A_{1}$.

For each $\theta \in(0,1)$ and $q \in[1, \infty)$, the Banach space $\left(A_{0}, A_{1}\right)_{\theta, q}$ consists of those elements $a \in A_{0}+A_{1}$ for which the norm

$$
\|a\|_{\left(A_{0}, A_{1}\right)_{\theta, q}}:=\left(\int_{0}^{\infty}\left(t^{-\theta} K\left(t, a ; A_{0}, A_{1}\right)\right)^{q} \frac{d t}{t}\right)^{1 / q}
$$

is finite. This definition extends to the case $q=\infty$ with

$$
\|a\|_{\left(A_{0}, A_{1}\right)_{\theta, \infty}}:=\sup _{t>0} t^{-\theta} K\left(t, a ; A_{0}, A_{1}\right) .
$$

Among the many known properties of these spaces, we mention the inclusions

$$
\left(A_{0}, A_{1}\right)_{\theta, q_{0}} \subset\left(A_{0}, A_{1}\right)_{\theta, q_{1}} \text { for all } \theta \in(0,1) \text { and } 1 \leq q_{0} \leq q_{1} \leq \infty
$$

whose proof can be found, e.g., in [1] p. 216 Corollary 7.17, [5] p. 46 or [41] pp. 25-26.

The complex interpolation method (A.P. Calderón [9]) Let $\left(A_{0}, A_{1}\right)$ be a Banach couple. Let $\mathcal{F}=\mathcal{F}\left(A_{0}, A_{1}\right)$ be the space of all functions $f$ of the complex variable $z=x+i y$ with values in $A_{0}+A_{1}$ that satisfy the following conditions:

(a) $f$ is continuous and bounded on the strip $0 \leq x \leq 1$ into $A_{0}+A_{1}$.

(b) $f$ is analytic from $0<0<1$ into $X_{0}+X_{1}$ (i.e., the derivative $f^{\prime}(z)$ exists in $A_{0}+A_{1}$ if $0<x=\operatorname{Re} z<1$ ). 
(c) $f$ is continuous on the line $x=0$ into $A_{0}$ and

$$
\|f(i y)\|_{X_{0}} \rightarrow 0 \quad \text { as }|y| \rightarrow \infty .
$$

(d) $f$ is continuous on the line $x=1$ into $A_{1}$ and

$$
\|f(1+i y)\|_{X_{1}} \rightarrow 0 \quad \text { as }|y| \rightarrow \infty .
$$

The space $\mathcal{F}$ is a Banach space with norm

$$
\|f\|_{\mathcal{F}}=\max \left\{\sup _{y \in \mathbb{R}}\|f(i y)\|_{X_{0}}, \sup _{y \in \mathbb{R}}\|f(1+i y)\|_{X_{1}}\right\} .
$$

Given a real number $\theta$ in the interval $(0,1)$, we define

$$
A_{\theta}=\left[A_{0}, A_{1}\right]_{\theta}=\left\{u \in A_{0}+A_{1}: u=f(\theta) \text { for some } f \in \mathcal{F}\right\} .
$$

The spaces $A_{\theta}$ are called complex interpolation spaces between $A_{0}$ and $A_{1}$; they are Banach spaces with respective norms

$$
\|u\|_{A_{\theta}}=\inf \left\{\|f\|_{\mathcal{F}}: f(\theta)=u\right\} .
$$

The basic interpolation theorem

Theorem 6.6 Let $\left(A_{0}, A_{1}\right)$ and $\left(B_{0}, B_{1}\right)$ be two Banach couples. If there exists a linear operator $T: A_{0}+A_{1} \rightarrow B_{0}+B_{1}$, which is continuous as a map from $A_{j}$ to $B_{j}$ for $j=0,1$, then, for each $\theta \in(0,1)$ and each $p \in[1, \infty]$,

$$
\|T\|_{\left(A_{0}, A_{1}\right)_{\theta, p} \rightarrow\left(B_{0}, B_{1}\right)_{\theta, p}} \leq\|T\|_{A_{0} \rightarrow B_{0}}^{\theta}\|T\|_{A_{1} \rightarrow B_{1}}^{1-\theta}
$$

and

$$
\|T\|_{\left[A_{0}, A_{1}\right]_{\theta} \rightarrow\left[B_{0}, B_{1}\right]_{\theta}} \leq\|T\|_{A_{0} \rightarrow B_{0}}^{\theta}\|T\|_{A_{1} \rightarrow B_{1}}^{1-\theta} .
$$

(See, e.g., [1] pp. 220-221, [5] pp. 40-41 and p. 88.)

The special case $A_{0}=A_{1}$ and some special norm estimates In the case where $A_{0}=A_{1}$ isometrically we obtain that $\left[A_{0}, A_{0}\right]_{\theta}=A_{0}$ for each $\theta \in(0,1)$, with

$$
\|a\|_{A_{0}}=\|a\|_{\left[A_{0}, A_{0}\right]_{\theta}}
$$

and $\left(A_{0}, A_{0}\right)_{\theta, p}=A_{0}$ for each $\theta \in(0,1)$ and $p \in[1, \infty]$, with

$$
\|a\|_{\left(A_{0}, A_{0}\right)_{\theta, p}}=c_{\theta, p}\|a\|_{A_{0}}
$$

where the constant $c_{\theta, p}$ is given by

$$
c_{\theta, \infty}=1 \quad \text { and } \quad c_{\theta, p}=\left(\frac{1}{\theta(1-\theta) p}\right)^{1 / p} .
$$


The proof of (6.11) is straightforward. For " $\leq$ " one can use the Phragmen-Lindelof theorem for analytic $A_{0}$ valued functions on the strip $0 \leq \theta \leq 1$. Then for " $\geq$ " one can use the function $f \in \mathcal{F}\left(A_{0}, A_{0}\right)$ defined by $f(z)=e^{\delta(z-\theta)^{2}} a$ where $\delta$ is an arbitrarily small positive number. The proof of (6.12) follows immediately from the fact that $K\left(t, a ; A_{0}, A_{0}\right)=\min \{1, t\}\|a\|_{A_{0}}$.

We will need some standard estimates for the norms $\|a\|_{\left[A_{0}, A_{1}\right]_{\theta}}$ and $\|a\|_{\left(A_{0}, A_{1}\right)_{\theta, p}}$ in the case where $a \in A_{0} \cap A_{1}$. These are

$$
\|a\|_{\left[A_{0}, A_{1}\right]_{\theta}} \leq\|a\|_{A_{0}}^{1-\theta}\|a\|_{A_{1}}^{\theta}
$$

and

$$
\|a\|_{\left(A_{0}, A_{1}\right)_{\theta, p}} \leq c_{\theta, p}\|a\|_{A_{0}}^{1-\theta}\|a\|_{A_{1}}^{\theta} .
$$

We can obtain (6.13) from an easy exercise using the function $f(z)=\frac{e^{\delta(z-\theta)^{2}}}{\|a\|_{A_{0}}^{1-z}\|a\|_{A_{1}}^{z}} a$ for arbitrarily small $\delta>0$ and an estimate very similar to (6.14) is implicit in pp. 49-50 of [5]. But we can also prove both (6.13) and (6.14) simultaneously, as follows. Let $X$ be either $\left(A_{0}, A_{1}\right)_{\theta, q}$ or $\left[A_{0}, A_{1}\right]_{\theta}$ and consider the linear operator $L: \mathbb{C} \rightarrow A_{0} \cap A_{1}$ defined by $L z=z a$ for each $z \in \mathbb{C}$. Then $\|L\| \mathbb{C} \rightarrow A_{j}=\|a\|_{A_{j}}$ for $j=0,1$ and so, by Theorem 6.6 and (6.11) and (6.12), we have

$$
\|a\|_{\left[A_{0}, A_{1}\right]_{\theta}}=\|L 1\|_{\left[A_{0}, A_{1}\right]_{\theta}} \leq\|a\|_{A_{0}}^{1-\theta}\|a\|_{A_{1}}^{\theta}\|1\|_{[\mathbb{C}, \mathbb{C}]_{\theta}}=\|a\|_{A_{0}}^{1-\theta}\|a\|_{A_{1}}^{\theta}
$$

and

$$
\|a\|_{\left(A_{0}, A_{1}\right)_{\theta, p}}=\|L 1\|_{\left(A_{0}, A_{1}\right)_{\theta, p}} \leq\|a\|_{A_{0}}^{1-\theta}\|a\|_{A_{1}}^{\theta}\|1\|(\mathbb{C}, \mathbb{C})_{\theta, p}=c_{\theta, p}\|a\|_{A_{0}}^{1-\theta}\|a\|_{A_{1}}^{\theta} .
$$

Interpolation formula for $L^{p}$ spaces When applied to a couple of $L^{p}$ spaces on the same underlying measure space, both the complex and the real methods (the latter for a suitable choice of the second parameter) yield an $L^{p}$ space with an intermediate exponent:

$$
\left(L^{p_{0}}, L^{p_{1}}\right)_{\theta, p}=\left[L^{p_{0}}, L^{p_{1}}\right]_{\theta}=L^{p} \quad \text { for all } 1 \leq p_{0}<p_{1} \leq \infty \text { and } \theta \in(0,1),
$$

where $p$ is given by $\frac{1}{p}=\frac{1-\theta}{p_{0}}+\frac{\theta}{p_{1}}$. (See e.g., [1] Corollary 7.27 p. 226 and Example 7.56 on pp. 249-250.)

Fractional Sobolev spaces and Besov spaces There are several equivalent definitions of these spaces. For our purposes here it will be convenient to define them via complex or real interpolation of Sobolev spaces and $L^{p}$ spaces.

Fractional Sobolev spaces can be equivalently defined (see e.g. [1] p. 250) by

$$
W^{s, p}\left(\mathbb{R}^{N}\right)=\left[W^{m, p}\left(\mathbb{R}^{N}\right), L^{p}\left(\mathbb{R}^{N}\right)\right]_{s / m}, \quad m \in \mathbb{N}, p \in(1, \infty), 0<s<m .
$$

Note that all choices of $m$ as above give the same space.

For each $s \in(0, \infty), p \in(1, \infty)$ and $q \in[1, \infty]$ the Besov space $B^{s, p, q}\left(\mathbb{R}^{N}\right)$ (see [41] p. 186, see also p. 230 of [1] as well as pp. 139-145 of [5]) can be defined 
by the formula:

$$
B^{s, p, q}\left(\mathbb{R}^{N}\right)=\left(W^{s_{0}, p}\left(\mathbb{R}^{N}\right), W^{s_{1}, p}\left(\mathbb{R}^{N}\right)\right)_{\theta, q}, \quad 0 \leq s_{0}<s<s_{1} \text { and } \theta=\frac{s-s_{0}}{s_{1}-s_{0}} .
$$

A commonly used version of this definition uses only integer values of $s_{0}$ and $s_{1}$. Analogously to the previous definition, all choices of $s_{0}$ and $s_{1}$ as above give the same space, to within equivalence of norms.

The Besov spaces satisfy the following continuous imbeddings (Jawerth [18], see also [41] Theorem 2.8 .1 p. 203):

$$
B^{s_{0}, p_{0}, q}\left(\mathbb{R}^{N}\right) \subset B^{s, p, q}\left(\mathbb{R}^{N}\right), \quad 1<p_{0}<p<\infty, 1 \leq q \leq \infty, s_{0}-s \geq n / p_{0}-n / p .
$$

These imbeddings can also be obtained from [1] Theorem 7.34 p. 231 by applying the reiteration formula for real interpolation spaces.

The Besov spaces also admit the following continuous imbeddings into $L^{p}$ spaces:

$$
B^{\{s, p, q\}}\left(\mathbb{R}^{N}\right) \subset L^{q}\left(\mathbb{R}^{N}\right), \quad s>0,1<p<\infty, p \leq q<p_{s}^{*} .
$$

For further background literature we refer to [28, 29] and [33].

\section{Appendix B: The iterated Brezis-Lieb lemma}

The following proposition evaluates the $L^{p}$-norms of sequences given by sums of terms with asymptotically disjoint supports. Although it and similar results have appeared elsewhere in literature, for the reader's convenience we explicitly recall its proof, which is an easy corollary of the well known Brezis-Lieb lemma [6].

Proposition 6.7 Suppose that $1 \leq p<\infty$. Let $y_{k}^{(n)}$ be a point in $\mathbb{R}^{N}$ for each $k$ and $n$ in $\mathbb{N}$. Suppose that $\lim _{k \rightarrow \infty}\left|y_{k}^{(m)}-y_{k}^{(n)}\right|=+\infty$ for each fixed $m$ and $n$ with $m \neq n$. Let $u_{k} \in L^{p}\left(\mathbb{R}^{n}\right)$ be a bounded sequence such that, for each $n \in \mathbb{N}$, the sequence $u_{k}\left(\cdot+y_{k}^{(n)}\right)$ converges weakly and almost everywhere to a function which we will denote by $w^{(n)}$. Then, for every $M \in \mathbb{N}$,

$$
\int_{\mathbb{R}^{N}}\left|u_{k}\right|^{p}-\sum_{n=1}^{M} \int_{\mathbb{R}^{N}}\left|w^{(n)}\right|^{p}-\int_{\mathbb{R}^{N}}\left|u_{k}-\sum_{n=1}^{M} w^{(n)}\left(\cdot-y_{k}^{(n)}\right)\right|^{p} \rightarrow 0 .
$$

Proof It of course suffices to show that every subsequence of the left side of (6.20) has itself a subsequence which tends to 0 as $k \rightarrow \infty$. This means that we can assume, without loss of generality, that the sequences $\left\{y_{k}^{(n)}\right\}_{k \in \mathbb{N}}$ satisfy the additional condition $\left|y_{k+1}^{(m)}-y_{k+1}^{(n)}\right|>2\left|y_{k}^{(m)}-y_{k}^{(n)}\right|$ for each $k \in \mathbb{N}$ and each $m, n \in\{1,2, \ldots, M\}$ with $m \neq n$. This guarantees that $\lim _{k \rightarrow \infty} f\left(x+y_{k}^{(m)}-y_{k}^{(n)}\right)=0$ for a.e. $x \in \mathbb{R}^{N}$ for each $f \in L^{p}\left(\mathbb{R}^{N}\right)$, and, in particular, whenever $f$ is any one of the functions $w^{(j)}$, $j \in \mathbb{N}$. 
We use induction. For $M=1$, the statement is immediate from the Brezis-Lieb lemma for the sequence $u_{k}\left(\cdot+y_{k}^{(1)}\right)$ whose weak and a.e. limit is $w^{(1)}$. Assume (6.20) is true for $M=m$ and let us show that it is true for $M=m+1$. Let

$$
v_{k}^{(m)}=u_{k}-\sum_{n=1}^{m} w^{(n)}\left(\cdot-y_{k}^{(n)}\right) .
$$

Applying the Brezis-Lieb lemma to the sequence $v_{k}^{(m)}\left(\cdot+y_{k}^{(m+1)}\right)$ whose weak and a.e. limit is $w^{(m+1)}$, we obtain from (6.20) the following:

$$
\begin{aligned}
0 & =\lim \left[\int_{\mathbb{R}^{N}}\left|u_{k}\right|^{p}-\sum_{n=1}^{m} \int_{\mathbb{R}^{N}}\left|w^{(n)}\right|^{p}-\int_{\mathbb{R}^{N}}\left|v_{k}^{(m)}\left(\cdot+y_{k}^{(n)}\right)\right|^{p}\right] \\
& =\lim \left[\int_{\mathbb{R}^{N}}\left|u_{k}\right|^{p}-\sum_{n=1}^{m} \int_{\mathbb{R}^{N}}\left|w^{(n)}\right|^{p}-\int_{\mathbb{R}^{N}}\left|w^{(m+1)}\right|^{p}-\int_{\mathbb{R}^{N}}\left|v_{k}^{(m+1)}\left(\cdot+y_{k}^{(n)}\right)\right|^{p}\right]
\end{aligned}
$$

which immediately gives (6.20) for $M=m+1$.

\section{References}

1. Adams, R., Fournier, J.: Sobolev Spaces, 2nd edn. Pure and Applied Mathematics, vol. 140. Academic Press, New York (2003)

2. Adimurthi Sandeep, K., Schindler, I., Tintarev, K. (eds.): Cocompact Imbeddings and Profile Decompositions, Proceedings of ICTS Program at TIFR-CAM, Bangalore, January 3-12 2012, Trends in Mathematics Sries. Birkhäuser (in preparation)

3. Bennett, C., Sharpley, R.: Interpolation of Operators. Academic Press, New York (1988)

4. Berestycki, H., Lions, P.-L.: Nonlinear scalar field equations I. Existence of a ground state. Arch. Ration. Mech. Anal. 82, 313-345 (1983)

5. Bergh, J., Löfström, J.: Interpolation Spaces. An Introduction. Grundlehren der mathematische Wissenschaften, vol. 223. Springer, Berlin (1976)

6. Brezis, H., Lieb, E.: A relation between pointwise convergence of functions and convergence of functionals. Proc. Am. Math. Soc. 88, 486-490 (1983)

7. Brudnyi, Ju.A., Krugljak, N.Ja.: Real Interpolation Functors. North-Holland, Amsterdam (1991)

8. Butzer, P.L., Berens, H.: Semi-groups of Operators and Approximation. Springer, Berlin (1967)

9. Calderón, A.P.: Intermediate spaces and interpolation, the complex method. Stud. Math. 24, 113-190 (1964)

10. Cobos, F., Kühn, T., Schonbek, T.: One-sided compactness results for Aronszajn-Gagliardo functors. J. Funct. Anal. 106, 274-313 (1992)

11. Cwikel, M.: Real and complex interpolation and extrapolation of compact operators. Duke Math. J. 65, 333-343 (1992)

12. Cwikel, M.: Complex interpolation of compact operators mapping into lattice couples. Proc. Est. Acad. Sci. 59, 19-28 (2010)

13. Cwikel, M., Janson, S.: Complex interpolation of compact operators mapping into the couple $\left(F L^{\infty}, F L_{1}^{\infty}\right)$. In: De Carli, L., Milman, M. (eds.) Contemporary Mathematics, vol. 445, pp. 71-92. American Mathematical Society, Providence (2007)

14. Cwikel, M., Kalton, N.J.: Interpolation of compact operators by the methods of Calderón and Gustavsson-Peetre. Proc. Edinb. Math. Soc. 38, 261-276 (1995)

15. Gérard, P.: Description du défaut de compacité de l'injection de Sobolev. ESAIM Control Optim. Calc. Var. 3, 213-233 (1998)

16. Holmstedt, T.: Interpolation of quasi-normed spaces. Math. Scand. 26, 177-190 (1970) 
17. Jaffard, S.: Analysis of the lack of compactness in the critical Sobolev embeddings. J. Funct. Anal. 161, 384-396 (1999)

18. Jawerth, B.: Some observations on Besov and Lizorkin-Triebel spaces. Math. Scand. 40, 94-104 (1977)

19. Koch, G.S.: Profile decompositions for critical Lebesgue and Besov space embeddings. arXiv:1006.3064

20. Lieb, E.: On the lowest eigenvalue of the Laplacian for the intersection of two domains. Invent. Math. 74, 441-448 (1983)

21. Lions, J.-L., Peetre, J.: Sur une classe d'espaces d'interpolation. Publ. Math. IHÉS 19, 5-68 (1964)

22. Lions, P.-L.: The concentration-compactness principle in the calculus of variations. The locally compact case, part 1. Ann. Inst. Henri Poincaré, Anal. Non Linéaire 1, 109-153 (1984)

23. Lions, P.-L.: The concentration-compactness principle in the calculus of variations. The locally compact case, part 2. Ann. Inst. Henri Poincaré, Anal. Non Linéaire 1, 223-283 (1984)

24. Lions, P.-L.: The concentration-compactness principle in the calculus of variations. The limit case, Part 1. Rev. Mat. Iberoam. 1(1), 145-201 (1985)

25. Lions, P.-L.: The concentration-compactness principle in the calculus of variations. The limit case, Part 2. Rev. Mat. Iberoam. 1(2), 45-121 (1985)

26. Lions, P.-L.: Solutions of Hartree-Fock equations for Coulomb systems. Commun. Math. Phys. 109, 33-97 (1987)

27. Peetre, J.: Espaces d'interpolation et théorème de Soboleff. Ann. Inst. Fourier 16(1), 279-317 (1966)

28. Peetre, J.: A theory of interpolation of normed spaces. Notas de Matematica No 39, Rio de Janeiro (1968), $88 \mathrm{pp}$

29. Peetre, J.: New Thoughts on Besov Spaces. Duke Univ. Math., vol. I. Duke Univ., Durham (1976)

30. Persson, A.: Compact linear mappings between interpolation spaces. Ark. Mat. 5, 215-219 (1964)

31. Schindler, I., Tintarev, K.: An abstract version of the concentration compactness principle. Rev. Mat. Complut. 15, 1-20 (2002)

32. Sickel, W., Skrzypczak, L.: Radial subspaces of Besov and Lizorkin-Triebel classes: extended Strauss lemma and compactness of imbeddings. J. Fourier Anal. Appl. 6, 639-662 (2000)

33. Sickel, W., Triebel, H.: Hölder inequality and sharp imbeddings in function spaces of $B_{p q}^{2}$ and $F_{p q}^{s}$ type. Z. Anal. Anwend. 14, 105-140 (1995)

34. Solimini, S.: A note on compactness-type properties with respect to Lorentz norms of bounded subsets of a Sobolev space. Ann. Inst. H. Poincaré, Sect. C 12, 319-337 (1995)

35. Strauss, W.A.: Existence of solitary waves in higher dimensions. Commun. Math. Phys. 55, 149-162 (1977)

36. Struwe, M.: Variational Methods: Applications to Nonlinear Partial Differential Equations and Hamiltonian Systems, 4th edn. A Series of Modern Surveys in Mathematics, vol. 34. Springer, Berlin (2008)

37. Tao, T.: A pseudoconformal compactification of the nonlinear Schrödinger equation and applications. N.Y. J. Math. 15, 265-282 (2009)

38. Tao, T., Visan, M., Zhang, X.: Minimal-mass blowup solutions of the mass-critical NLS. Forum Math. 20, 881-919 (2008)

39. Tintarev, K.: Cocompact imbeddings and structure of weakly convergent sequences. In: Maz'ya, V., Isakov, V. (eds.) Sobolev Spaces in Mathematics, Vol. 1: Sobolev Type Inequalities. International Mathematical Series, vol. 8, pp. 361-375. Springer, Berlin (2009)

40. Tintarev, K., Fieseler, H.: Concentration Compactness: Functional-Analytic Grounds and Applications. Imperial College Press, London (2007)

41. Triebel, H.: Interpolation Theory, Function Spaces, Differential Operators. North-Holland, Amsterdam (1978) 